\title{
ADAPTIVE NESTED TRELLIS DECODING FOR BLOCK CODES
}

\author{
Bahram Honary, Garegin Markarian and Michael Darnell \\ Bahram Honary, Professor, LancasterUniversity, Lancaster,UK \\ Garegin Markarian, Lecturer, LancasterUniversity்; Lancaster,UK \\ Michael Darnell, Professor, Hull University, Hull, UK
}

\begin{abstract}
A new adaptive trellis decoding technique for block codes is proposed. The technique is based on a recently introduced encoding technique which allows the design of almost all known linear codes together with their minimal trellises. The proposed adaptive trellis decoding technique allows the implementation of a single soft maximum likelihood trellis decoder for decoding of different codes. It has been shown that both the encoding and decoding techniques can be implemented with a low complexity and cost.
\end{abstract}

\section{INTRODUCTION.}

Trellis decoding technique for linear block codes has received sufficient attention for both practical and theoretical reasons [1-5]. On the practical side, a minimal trellis structure of a block code can be used for performing lowcomplexity maximum likelihood soft decision decoding. From the theoretical viewpoint, such trellises can be used for constructing other codes. In this paper we introduce a new simple encoding technique which allows the design of almost all known linear block codes together with their trellises. The trellises of the designed codes are similar to the trellises of the coset codes introduced by Forney [2]. However, the encoding procedure is simpler and allows the design of linear block codes with any arbitrary code length.

\section{CODE DESIGN TECHNIQUE.}

The technique is based on generalised array codes (GACs) and their trellis structures [6]. A generalised $\left(n, k, d_{\text {min }}\right)$ array code is an array code in which the column and row subcodes may have different numbers of information and parity check symbols; the code length $n=n_{1} n_{2}$ and the total number of information digits, $\mathrm{k}=\mathrm{k}_{1}+\mathrm{k}_{2}+\ldots+\mathrm{k}_{\mathrm{n} 2}$, where $\mathrm{n}_{1}$ and $\mathrm{n}_{2}$ are the number of columns and rows respectively, and $k_{p}$ is the number of information digits in the pth row [6]. GACs may be considered as being formed from the union of coset(s) of a basis conventional array code, where the cosets are constructed such that the union of cosets has the same minimum distance as that of the parent array code. Using the concept of the GAC, almost all known linear block codes together with their low complexity trellises can be designed. The encoding procedure can be also described as an array representation of coset codes introduced by Forney [2]. To illustrate the proposed technique an example is given here for an $(8,4,4)$ GAC which is equivalent to the well known $(8,4,4)$ Reed-Muller (RM) code. The procedure for code design is given below:

(i) Design the basic, $(8,3,4),\left(n=4 \times 2, n_{1}=2\right.$, $\mathrm{n}_{2}=4$ ) array code, $\mathrm{C}_{1}$, with the single-parity check $(4,3,2)$ column and repetition row codes $\mathrm{R}_{1}=(2,1,2)$ :

$$
c_{1}=\begin{aligned}
& x_{1} p_{1} \\
& x_{2} p_{2} \\
& x_{3} p_{3} \\
& p_{4} p_{4}
\end{aligned}
$$

where $x_{i}, i=1,2,3$, represent information digits and $p_{j}, j=1, \ldots 4$, represent parity check symbols. (ii) Design an additional array code, $\mathrm{C}_{2}$, with the following structure: 


$$
C_{2}=\begin{array}{ll}
0 & x_{4} \\
0 & x_{4} \\
0 & x_{4} \\
0 & x_{4}
\end{array}
$$

where $x_{4}$ is an additional information digit. (iii) Add the two codes $\mathrm{C}_{1}$ and $\mathrm{C}_{2}$ on a modulo-2 basis as follows:

$$
C=C_{1} \oplus C_{2}=\begin{array}{ll}
x_{1} & \left(x_{4} \oplus p_{1}\right) \\
x_{2} & \left(x_{4} \oplus p_{2}\right) \\
x_{3} & \left(x_{4} \oplus p_{3}\right) \\
p_{4} & \left(x_{4} \oplus p_{4}\right)
\end{array}
$$

The designed code is a linear non-systematic code and has the following parameters: $\left(\mathrm{n}_{0}=8, \mathrm{k}_{0}=4, \mathrm{~d}_{0}=4\right)$. It has been shown [7] that by deleting the symbol which is located in the second column and 4 th row, the designed $(8,4,4)$ code will be transformed to the $(7,4,3)$ Hamming code.

The proposed technique has been investigated for the design of other known codes, such as $(15,5,7) \mathrm{BCH},(16,5,8) \mathrm{RM}$, $(15,7,5) \quad \mathrm{BCH}, \quad(17,9,5)$ optimum, $(24,12,8)$ extended Golay, $(23,12,7)$ Golay, etc. For example, following the procedure outlined above, one can design the $(32,6,16)$ RM binary code by choosing a $(4 \times 8)\left(n_{1}=4, n_{2}=8\right)$ basic array code, $C_{1}$, with an $(8,1,8)$ row code $R_{1}$, and $(4,3,2)$ single parity check column code. The first additional code, $\mathrm{C}_{2}$, should consist of a (4 $x 5)$ all zero matrix and a $(4 \times 3)$ matrix of repetition column codes. The $(31,6,15)$ code can be derived easily by deleting the parity check symbol which is located in the 4th row and 8th column. Figure 1 illustrates the trellis diagrams of a few examples of the above codes. It is apparent from this Figure that the proposed code design technique provides codes with very low-complexity trellis structures.

\section{NESTED TRELLIS DECODING.}

In many practical applications, such as timevarying communication channels, it is vital to have an adaptive coding scheme in which different elements of the overall coding format have different level of resilience to noise and distortion. The conventional adaptive block coding techniques use a number of different encoders/decoders each for different channel conditions. Although the error performance of such systems is improved, their complexity and cost are increased dramatically. To reduce the cost of such a system it is vital to have a single soft maximum likelihood trellis decoder (SMLTD) which could deal with different codes being used for information transmission; however, known encoding/decoding techniques do not posses such properties.

In this paper a novel nested soft maximum likelihood trellis decoding (SMLTD) SMLTD technique is introduced [9]. The technique can be employed for decoding a number of different block codes in single decoder, and provides low complexity implementation in comparison with other techniques.

A new adaptive trellis decoding technique for product codes based on GACs has been introduced recently [6]. This adaptive coding and SMLTD scheme allows us to use one encoder/decoder for different codes. This may be done either by designing a set of codes that have equal number of states and columns in their trellises and only differ in labelling of trellis branches [7-9] or alternately by designing a set of different codes with trellises which are embedded into each other. The trellises of such codes will be nested into each other and the procedure for the design of such an adaptive encoder and its SMLTD is as follows.

(i) Lèt $(n, k, d)$ be a block code with maximal code length and information rate.

(ii) Represent $\mathrm{n}=\mathrm{m} \times \mathrm{n}_{2}$ where both $\mathrm{m}$ and $\mathrm{n}_{2}$ are integers and define the size of array ( $\mathrm{m}$ is number of columns). The following condition

$$
d \geq n_{2}
$$

must be satisfied in order to design the best code, where $d$ is a minimum Hamming distance of the code.

(iii) Design a basic ( $\left.m \times n_{2}, \mathrm{k}, \mathrm{d}\right)$ GAC [6];

(iv) The trellis diagram of the designed GAC will have $\mathrm{N}_{\mathrm{S}}=2^{\{\text {maxkp\} }}$ number of states and $\mathrm{N}_{\mathrm{C}}=\mathrm{m}+1$ number of columns, where $k_{p}$ is number of information digits in $p$-th row, and each branch will be labelled with $\mathrm{n}_{2}$ digits according to [6]. 
(v) Delete the parity check symbol located in mth row and $n_{2}$ nd column. A new code will have the following parameters (n$1, \mathrm{k}, \mathrm{d}-1)$ and a similar trellis. The only difference between two trellises will be in the number of digits being used for labelling branches at final depth of the trellis.

(vi) Delete the pth column in the basic GAC. A new code will have the following parameters $\left(n-n_{2}, k-1, d^{\prime}\right)$, where $d^{\prime} \geq n_{2}-1$. The trellis diagram for the new code will have the same number of columns $\mathrm{N}_{\mathrm{C}}$, but number of states will be reduced to $\mathrm{N}_{\mathrm{c}}=2^{\max \{\mathrm{kp}-1\}}$. The labelling of the branches will require $n_{2}-1$ digits and the new trellis is a part of the basic trellis.

(vii) Delete the parity check symbol located in mth row and $n_{2}-1$ column. The new code will have the following parameters $\left(\mathrm{n}-\mathrm{n}_{2}-1, \mathrm{k}-1, \mathrm{~d}^{\prime}-1\right)$ and a similar trellis. The only difference between two trellises will be in the number of digits being used for labelling branches at final depth of the trellis.

(viii) Repeat steps (vi) and (vii) until only one information digit will be left (repetition code). In this case $\mathrm{N}_{\mathrm{S}}=2$ and $\mathrm{N}_{\mathrm{c}}=\mathrm{m}+1$.

An example of such a novel technique is shown in Fig.2. From this Figure it follows that trellises of different codes $((4,1,4)$ repetition [2], $(4,3,2)$ single parity check [2], $(7,4,3)$ Hamming [7], $(8,4,4)$ Reed-Muller [7], $(15,5,7)$ $\mathrm{BCH}[9],(16,5,8)$ Reed-Muller [9], $(31,6,15) \mathrm{BCH}$ [9]) are nested into the trellis diagram of the $(32,6,16)$ Reed-Muller code [9], represented in the form of GAC. This means, that the presented trellis diagram can be used for soft maximum likelihood decoding of any of the above listed codes. The trellis diagram shown in Figure 2 can be nested itself into a trellis of block codes with higher block lengths or information rates. For example, all the trellises shown in Figure 2 can be nested into the trellis diagram of $(32,20,8)$ block code which has the same number of columns and 64 states.

The information throughput of the system can be optimised by an appropriate choice of component codes. This can be achieved since the size of nested trellis (number of states and columns) depends on the component codes.

\section{CONCLUSION}

A novel low complexity adaptive nested SMLTD technique is introduced. The technique is applicable in all communication systems aiming at providing low-complexity encoding and soft maximum likelihood trellis decoding of block binary error control codes. The main benefit would be lower complexity (and hence lower cost) and maximum likelihood performance.

\section{REFERENCES}

1. Wolf J.K. "Efficient maximum likelihood decoding of linear block codes using a trellis". IEEE Transactions on Information Theory, vol.IT-24, No 1 , 1978, pp.76-80.

2. Forney G.D. "Coset codes-Part 2: Binary lattices and related codes". IEEE Transactions on Information Theory, vol.IT-34, No 5, 1988, pp. 1152-1187.

3. Muder D.J. "Minimal trellises for block codes". IEEE Transactions on Information Theory, vol.IT-34, No 5, 1988, pp.10491053.

4. Kasami T., Takata T., Fujiwara T., S.Lin. "On complexity of trellis structure of linear block codes". Proc. of IEEE International Symposium on Information Theory, Budapest, Hungary, June 1991.

5. Berger Y., Be'ery Y. Bounds of the Trellis Size of Linear Block Codes. "IEEE Transactions on Information Theory", vol.39, No 1, 1993, pp.203-209.

6. Honary B., Markarian G., Farrell P. "Generalised array codes and their trellis structure". Electronics Letters, vol.29, No 6, 1993, pp.541-542.

7. Honary B., Markarian G. "Low complexity trellis decoding of Hamming codes". Electronics Letters, vol.29, No 12, 1993, pp.1114-1116.

8. Markarian G., Honary B., Soyjaudah K.M. "Trellis decoding technique of adaptive product codes".- Proceedings of 2nd International Symposium on Communication Theory and Applications", July 1993, Lake District, UK, p.96.

9. Honary B., Markarian G, Darnell M. Nested Trellis Decoder For Block Codes. UK Patent Application No.9414276-7, 14.07.94. 


\section{Fig.1. Trellises of Different Block Codes}

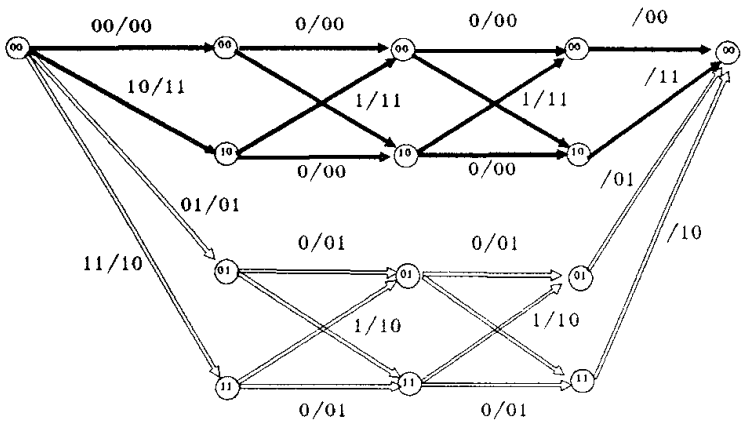

Trellis Diagram of the $(8,4,4)$ RM Code

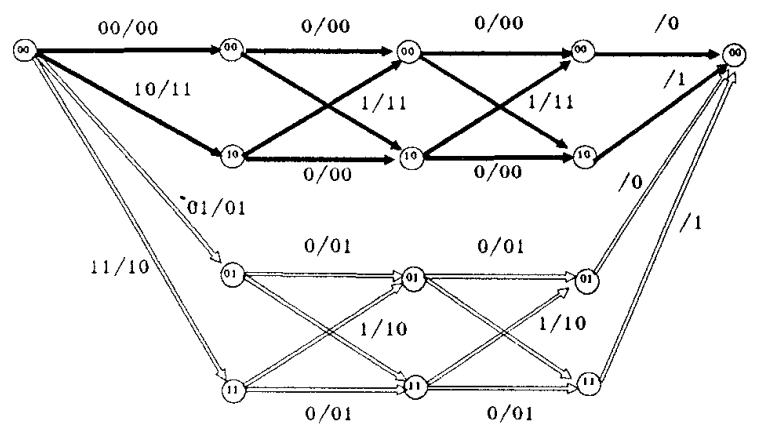

Trellis Diagram of the $(7,4,3)$ Hamming Code

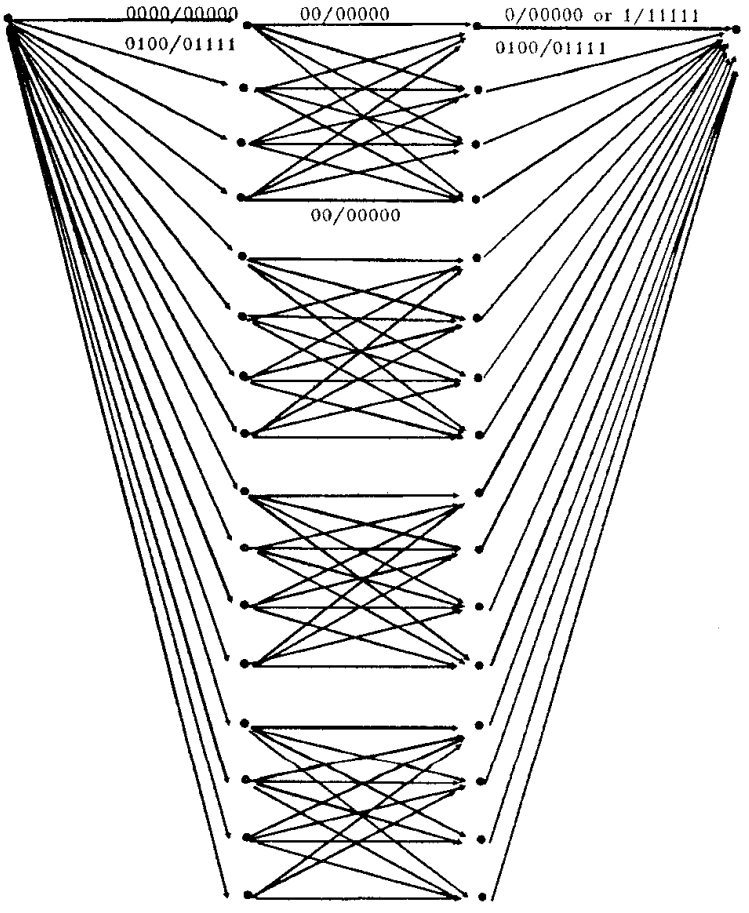

Trellis Diagram of the $(15,7,5)$ GAC

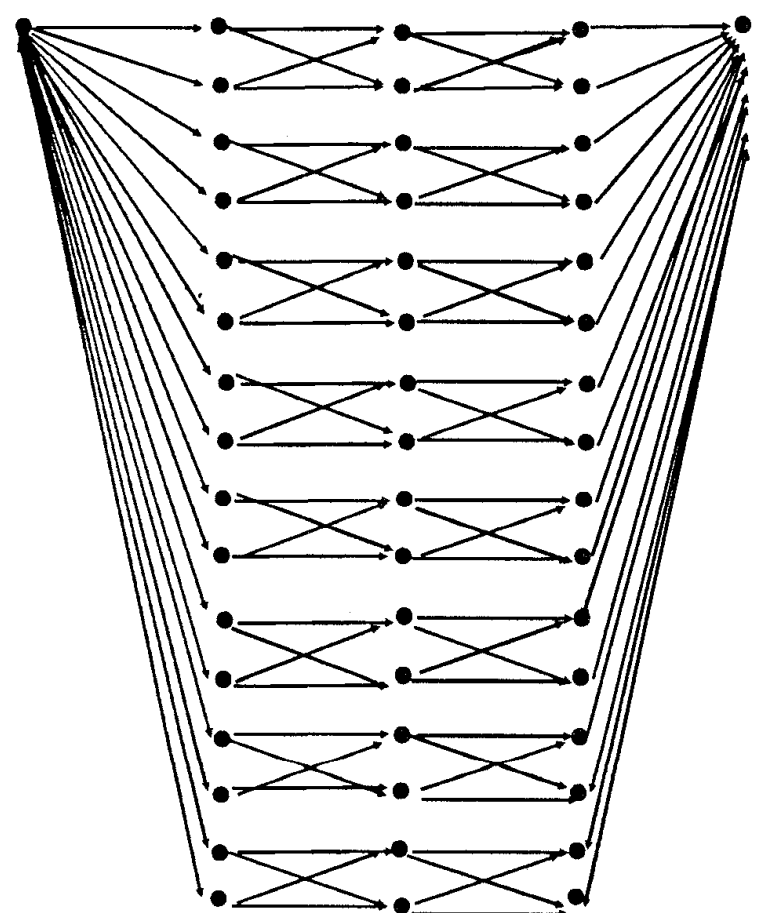

Trellis Diagram of the $(31,6,15)$ Code 


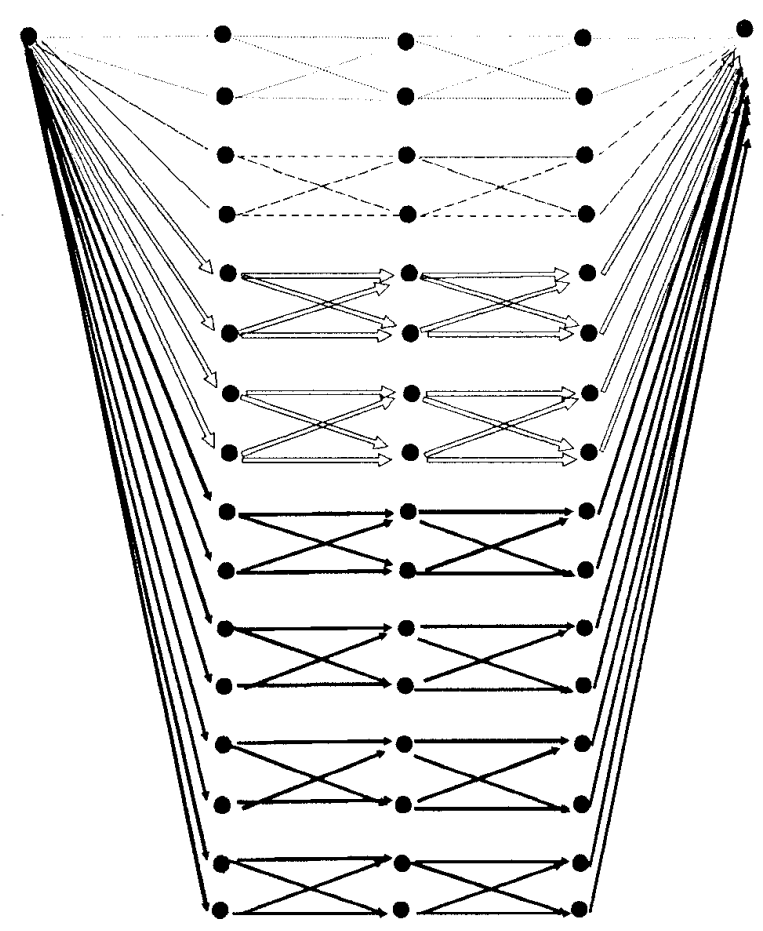

Trellis No 1 , for $(4,3,2)$ code.

Trellis No 2 together with trellis No 1, for both $(7,4,3$,$) Hamming and (8,4,4)$ Reed-Muller codes.

Trellis No 3, together with NoNa 1 and 2 for both $(15,5,7) \mathrm{BCH}$ and $(16,5,8) \mathrm{RM}$ codes.

Treelis No 4, together with NoNo 1,2 , and 3 for both $(31,6,15) \mathrm{BCH}$ and $(32,6,16) \mathrm{RM}$ codes.

\section{Fig.2. Trellis Diagram of the Adaptive Nested Trellis Decoder}

